\title{
Reference signal extraction under a noisy environment for a semi-adaptive sound reproduction system
}

\author{
Yosuke Tatekura ${ }^{a)}$ and Norihiro Yoshida \\ Faculty of Engineering, Shizuoka University \\ 3-5-1 Johoku, Naka-ku, Hamamatsu, Shizuoka 432-8561, Japan \\ a) tytatek@ipc.shizuoka.ac.jp
}

\begin{abstract}
In the present paper, we propose a method of reference signal extraction for a semi-adaptive sound reproduction system based on semi-blind source separation under a noisy environment. Since the fluctuation of room transfer functions degrades the reproduced sound, we have proposed a semi-adaptive sound reproduction system that updates the inverse filters. However, in a noisy environment, it is difficult to observe only the reference signal. Therefore, we use semi-blind source separation to obtain a clean reference signal from a noisy observed signal. The simulation results reveal that the proposed method can obtain the reference signal with high accuracy.
\end{abstract}

Keywords: multichannel sound reproduction system, semi-adaptation, semi-blind source separation, noise reduction

Classification: Science and engineering for electronics

\section{References}

[1] J. Bauck and D. H. Cooper, "Generalized transaural stereo and applications," J. Audio Eng. Soc., vol. 44, no. 9, pp. 683-705, 1996.

[2] Y. Yai, S. Miyabe, H. Saruwatari, K. Shikano, and Y. Tatekura, "Rapid compensation of temperature fluctuation effect for multichannel sound field reproduction system," IEICE Trans. Fundamentals, vol. E91-A, no. 6, pp. 1329-1336, 2008.

[3] Y. Tatekura, H. Saruwatari, and K. Shikano, "Sound reproduction system including adaptive compensation of temperature fluctuation effect for broad-band sound control," IEICE Trans. Fundamentals, vol. E85-A, no. 8, pp. 1851-1860, 2002.

[4] J. Even, H. Saruwatari, and K. Shikano, "Frequency domain semi-blind signal separation: Application to the rejection of internal noises," Proc. ICASSP2008, Las Vegas, U.S.A., pp. 157-160, April 2008.

[5] N. Murata and S. Ikeda, "An on-line algorithm for blind source separation on speech signals," Proc. NOLTA98, Crans-Montana, Switzerland, pp. 923-926, Sept. 1998.

[6] N. Aoshima, "Computer-generated pulse signal applied for sound measurement," J. Acoust. Soc. Am., vol. 69, pp. 1484-1488, 1981. 


\section{Introduction}

In order to realize a sound reproduction system with several loudspeakers, inverse filters are designed and used to cancel the effect of room transfer functions (RTFs) [1]. However, RTFs are not invariant and vary with the environmental conditions, such as temperature. Therefore, in sound reproduction systems that use fixed inverse filters, reproduction accuracy is degraded by fluctuations in the environment. We previously proposed a semi-adaptive sound reproduction system that compensates for environmental fluctuations, such as temperature fluctuations [2], and an inverse filter relaxation algorithm [3] in order to maintain the quality of the reproduced sound. In this system, one monitoring microphone can be placed at a location that does not restrict the listener, and the inverse filters can be updated by the signal observed at the monitoring microphone as a reference signal. However, since the environment normally contains several noise sources, it is difficult to observe only the reproduced signal by the conventional system.

To resolve this problem, we herein propose a method for observing only the reproduced signal in a noisy environment using the semi-adaptive sound reproduction system. In the proposed method, we introduce semi-blind source separation (semi-BSS) [4] to the semi-adaptive sound reproduction system for extraction of the reproduced signal from the noisy observed signal.

\section{Semi-adaptive sound reproduction system}

\subsection{Sound reproduction system with inverse filtering}

In the following, a multichannel sound reproduction system with $M$ loudspeakers as secondary sound sources $\mathrm{L}_{m}(m=1,2, \cdots, M)$ and $N$ control points $\mathrm{C}_{n}(n=1,2, \cdots, N)$ is assumed. The matrix representing the RTF $G_{n m}(\omega)$ is denoted as $\mathbf{G}(\omega)=\left[G_{n m}(\omega)\right]_{n m}$, and the matrix representing the inverse filter $H_{m n}(\omega)$ is denoted as $\mathbf{H}(\omega)=\left[H_{m n}(\omega)\right]_{m n}$. In addition, $[a]_{i j}$ is a matrix that has an element $a$ in the $i$-th row and $j$-th column, and $\omega$ denotes the frequency. The vector representing the original source signal is denoted as $\mathbf{D}(\omega)=\left[D_{1}(\omega), \cdots, D_{N}(\omega)\right]^{T}$, and the vector representing the reproduced sound signal is denoted as $\mathbf{X}(\omega)=\left[X_{1}(\omega), \cdots, X_{N}(\omega)\right]^{T}$, where $D_{n}(\omega)$ is the original sound signal reproduced at the $n$-th control point and $X_{n}(\omega)$ is the reproduced sound signal at the $n$-th control point. The reproduced signal $\mathbf{X}(\omega)$ can be expressed in terms of the above matrices as follows:

$$
\mathbf{X}(\omega)=\mathbf{G}(\omega) \mathbf{H}(\omega) \mathbf{D}(\omega) .
$$

Since our objective is to achieve control such that $\mathbf{X}(\omega)=\mathbf{D}(\omega), \mathbf{H}(\omega)$ can be derived as the generalized inverse matrix of $\mathbf{G}(\omega)$ in the case of $M>N$. Since the solution becomes underdetermined if there is no rank reduction, the generalized Moore-Penrose (MP) inverse matrix with the least norm solution (LNS) is used. In order to derive the generalized MP inverse matrix of $\mathbf{G}(\omega)$ as $\mathbf{H}(\omega)$, the singular value decomposition of $\mathbf{G}(\omega)$ is carried out for each $\omega$. 


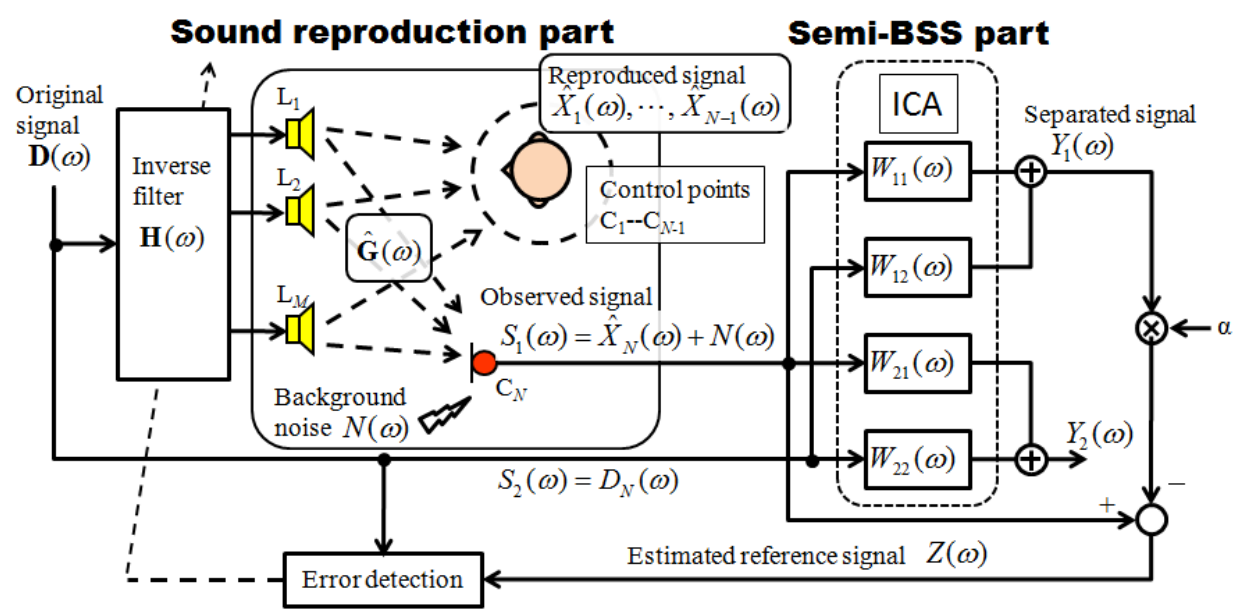

Fig. 1. Extraction of the reproduced signal from the noisy observed signal by semi-BSS in the semi-adaptive sound reproduction system.

\subsection{Semi-adaptation and its problem}

For the cases in which the RTFs do not fluctuate, the observed signal $\mathbf{X}(\omega)$ can be written as Eq. (1). However, the RTFs are not time invariant and vary with fluctuations in the environment. If $\hat{\mathbf{G}}(\omega)$ represents the RTFs obtained after the fluctuation of $\mathbf{G}(\omega)$, then $\hat{\mathbf{G}}(\omega)$ is given by

$$
\hat{\mathbf{G}}(\omega)=\mathbf{G}(\omega)+\Delta \mathbf{G}(\omega),
$$

where $\Delta \mathbf{G}(\omega)=\left[\Delta G_{n m}(\omega)\right]_{n m}$ expresses the differential of $\mathbf{G}(\omega)$ and $\hat{\mathbf{G}}(\omega)$. Here, the observed signal after fluctuation $\hat{\mathbf{X}}(\omega)$ can be expressed as

$$
\begin{aligned}
\hat{\mathbf{X}}(\omega) & =\hat{\mathbf{G}}(\omega) \mathbf{H}(\omega) \mathbf{D}(\omega) \\
& =\mathbf{D}(\omega)+\Delta \mathbf{G}(\omega) \mathbf{H}(\omega) \mathbf{D}(\omega) .
\end{aligned}
$$

The left-hand side of Fig. 1 shows an overview of a semi-adaptive sound reproduction system. The monitoring microphone $\mathrm{C}_{N}$ is set apart from the listener so as to promote agreeable listening. The observed signal after fluctuation $\hat{X}_{N}(\omega)$ observed at the monitoring microphone $\mathrm{C}_{N}$ is also given by

$$
\hat{X}_{N}(\omega)=D_{N}(\omega)+\Delta \mathbf{G}_{N}(\omega) \mathbf{H}(\omega) \mathbf{D}(\omega),
$$

where $\Delta \mathbf{G}_{N}=\left[\Delta G_{N 1}(\omega), \Delta G_{N 2}(\omega), \cdots, \Delta G_{N M}(\omega)\right]$ represents the RTFs from each loudspeaker to the monitoring microphone. Using the observed signal $\hat{X}_{N}(\omega)$ as the reference signal, all of the inverse filters can be updated $[2,3]$.

However, these semi-adaptive algorithms are assumed to detect only the reproduced sound from the system by the monitoring microphone. If there are any noise sources inside or outside the reproduced room, the monitoring microphone detects $\hat{X}_{N}(\omega)+N(\omega)$ rather than $\hat{X}_{N}(\omega)$, where $N(\omega)$ is the noise signal. As such, when several background noises, such as the voice of the user, are also observed, the algorithms do not necessarily work correctly. Therefore, we must extract only the reproduced signal $\hat{X}_{N}(\omega)$ from $\hat{X}_{N}(\omega)+$ $N(\omega)$. 


\section{Reference signal extraction by semi-BSS}

\subsection{Semi-BSS based on FDICA}

Blind source separation (BSS) based on frequency domain independent component analysis (FDICA) is a technique for estimating an original sound source based solely on the mixed signals observed at each microphone [5]. This technique does not necessarily require prior information such as the direction of the target sound source or speech break. If one of original sound sources is known, then conventional FDICA-based BSS can be developed into semi-BSS [4]. In the proposed semi-adaptive sound reproduction system, we introduce the semi-BSS method to obtain the reference signal from a noisy observed signal.

The separated signal of semi-BSS, i.e., $\mathbf{Y}(\omega)=\left[Y_{1}(\omega), Y_{2}(\omega)\right]^{T}$, can be written in terms of the $2 \times 2$ separation matrix $\mathbf{W}(\omega)$ and the input signal $\mathbf{S}(\omega)=\left[S_{1}(\omega), S_{2}(\omega)\right]^{T}$ as follows:

$$
\mathbf{Y}(\omega)=\mathbf{W}(\omega) \mathbf{S}(\omega)
$$

In semi-BSS, the component $S_{2}(\omega)$ of $\mathbf{S}(\omega)$ is assumed to be a known signal, and so we can set $S_{2}(\omega)=D_{N}(\omega)$. The input signal $S_{1}(\omega)$ is set to be the observed signal at the monitoring microphone, which can be written as $S_{1}(\omega)=\hat{X}_{N}(\omega)+N(\omega)$. We can write $\mathbf{W}(\omega)$ in semi-BSS as follows:

$$
\mathbf{W}(\omega)=\left[\begin{array}{cc}
W_{11}(\omega) & W_{12}(\omega) \\
0 & W_{22}(\omega)
\end{array}\right]
$$

Here, $\mathbf{W}(\omega)$ is optimized with the following iterative update procedure:

$$
\mathbf{W}_{i+1}(\omega)=\mathbf{W}_{i}(\omega)+\mu\left(\mathbf{I}-E\left[\varphi(\mathbf{Y}(\omega)) \mathbf{Y}(\omega)^{T}\right]\right) \mathbf{W}_{i}(\omega),
$$

where $i$ is the iteration time, $\mu$ is the step size parameter, $\mathbf{I}$ is the $2 \times 2$ identity matrix, and $\varphi(\cdot)$ is a nonlinear vector function.

Note that, in actual BSS, the short-time analysis of the input signals is performed using DFT in a frame-by-frame manner.

\subsection{Reference signal extraction from the noisy observed sig- nal}

The reproduced signal picked up by the monitoring microphone $X_{N}(\omega)$ is expected to be equivalent to the original sound source signal $D_{N}(\omega)$ in the absence of environmental fluctuation. Although the RTFs fluctuated as shown in Eq. (2), the original signal $D_{N}(\omega)$ and the fluctuated reproduced signal $\hat{X}_{N}(\omega)$ are highly similarity. Therefore, $\hat{X}_{N}(\omega)$ can be separated with high accuracy from noisy observed signal $S_{1}(\omega)$ using $D_{N}(\omega)$ by semi-BSS. However, semi-BSS extracts an unknown signal from the observed signal using a known signal. In other words, the noise signal, and not the reproduced signal, can be obtained directly. The goal is to obtain $\hat{X}_{N}(\omega)$ from noisy observed signal $S_{1}(\omega)$. 


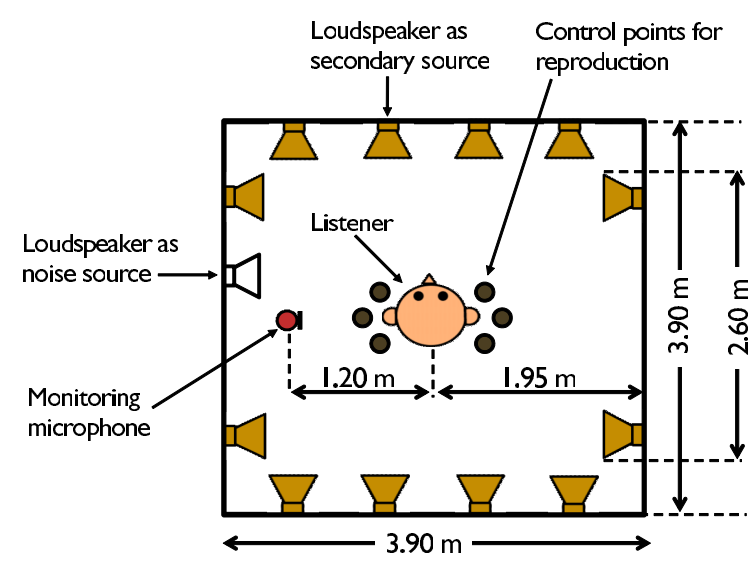

Fig. 2. Experimental setup.

Therefore, we first obtain the noise signal $N(\omega)$ from $S_{1}(\omega)$ by semi-BSS. Next, the estimated reference signal $Z(\omega)$, which is assumed to be $\hat{X}_{N}(\omega)$, is obtained by subtracting the noise signal from the observed signal, as follows:

$$
Z(\omega)=S_{1}(\omega)-\alpha Y_{1}(\omega)
$$

where $\alpha$ represents the scaling parameter. An optimized $\alpha, \hat{\alpha}$, is obtained in order to minimize the spectrum distortion (SD) between $Z(\omega)$ and the original source signal $D_{N}(\omega)$ :

$$
\begin{aligned}
\hat{\alpha} & =\underset{\alpha}{\operatorname{argmin}} \sqrt{\frac{1}{N} \sum_{\omega}\left(20 \log \frac{|Z(\omega)|}{\left|D_{N}(\omega)\right|}\right)^{2}} \\
& =\underset{\alpha}{\operatorname{argmin}} \sqrt{\frac{1}{N} \sum_{\omega}\left(20 \log \frac{\left|S_{1}(\omega)-\alpha Y_{1}(\omega)\right|}{\left|D_{N}(\omega)\right|}\right)^{2}} .
\end{aligned}
$$

\section{Numerical simulation}

\subsection{Numerical condition}

A sound reproduction system having 12 loudspeakers and seven control points was set up in the experimental room, in which the reverberation is approximately 0.14 seconds. The arrangement is shown in Fig. 2 .

The impulse responses of the RTFs used in this simulation are obtained by a time stretched pulse signal [6] of 65,536 points, where the sampling frequency is $48,000 \mathrm{~Hz}$, the quantization is $16 \mathrm{bits}$, and averaging is performed four times. In this simulation, these impulse responses are downsampled from $48,000 \mathrm{~Hz}$ to $8,000 \mathrm{~Hz}$. The fluctuation of the RTFs is assumed to be caused by temperature variation in the room, and the impulse responses were measured at several temperatures. The inverse filters are designed by the MP generalized inverse matrix of $\mathbf{G}(\omega)$ under the initial temperature condition. A music signal of 9 seconds is used as the original signal for reproduction, and different music signal is used as the original signal for monitoring. Three signals, namely, a telephone ring tone, a clock alarm, and a speech signal were used for background noise. 


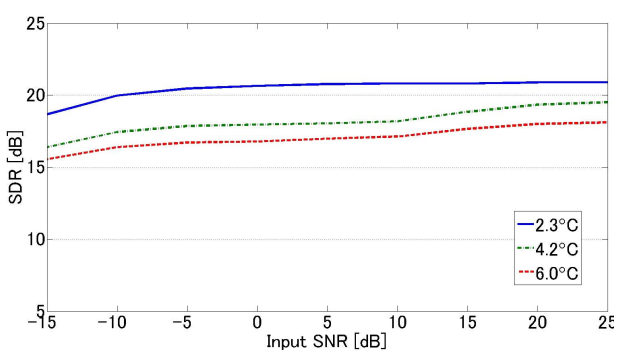

(a)

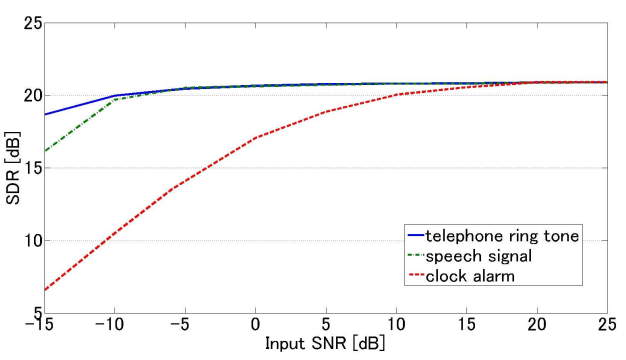

(b)

Fig. 3. Signal to deviation ratio of the extracted signal for different input SNR conditions for (a) different temperature fluctuations and (b) background noise conditions.

As the analysis conditions of semi-BSS, the frame length was $256 \mathrm{~ms}$, and the frame shift was $64 \mathrm{~ms}$. In Eq. (7), the tanh function is used as the nonlinear function. The iteration times and step size for which the extraction accuracy is maximum are set by preliminary experiments at each input SNR.

\subsection{Experimental results}

Figure 3 (a) shows the result for extraction accuracy under different temperature fluctuation conditions using the telephone ring tone as the background noise signal. The signal to deviation ratio (SDR) is used to evaluate the extraction accuracy, which can be computed as follows:

$$
\operatorname{SDR}[\mathrm{dB}]=10 \log _{10} \frac{\sum_{n}|z(n)|^{2}}{\sum_{n}\left|z(n)-\hat{x}_{N}(n)\right|^{2}},
$$

where $z(n)$ and $\hat{x}_{N}(n)$ are time domain representations of $Z(\omega)$ and $\hat{X}_{N}(\omega)$, respectively. This result shows that high extraction accuracies are maintained under all of the temperature fluctuation conditions.

Figure 3 (b) shows the results for extraction accuracy for different background noises where the temperature fluctuation is $2.3^{\circ} \mathrm{C}$. The figure indicates large differences in the extraction accuracy depending on the type of background noise at lower input SNRs. However, an extraction accuracy of over $15 \mathrm{~dB}$ can be obtained under any condition using the proposed method considered herein when the input SNR exceeds $0 \mathrm{~dB}$.

\section{Conclusion}

We have presented an algorithm to extract a reference signal from a noisy observed signal for the semi-adaptive sound reproduction system by semiBSS. In a simulation using real environmental data, the proposed method was demonstrated to extract the reference signal with a high SDR. In addition, the results reveal that the proposed method is robust against temperature fluctuation of the reproduced room and various types of noise. In future studies, we intend to apply the proposed method to an actual semi-adaptive sound reproduction system and evaluate its efficiency. 